\title{
Erratum to: Stochastic optimization of a biologically plausible spino-neuromuscular system model
}

\section{A comparison with human subjects}

\author{
Stanley Gotshall • Kathy Browder • \\ Jessica Sampson $\cdot$ Terence Soule $\cdot$ Richard Wells
}

Published online: 17 June 2010

(C) Springer Science+Business Media, LLC 2010

\section{Erratum to: Genet Program Evolvable Mach (2007) 8:355-380 DOI 10.1007/s10710-007-9044-8}

There were errors in Fig. 11, Eq. 5, and section 4.4 in the final manuscript published in Genetic Programming and Evolvable Machines (2007) 8:355-380. The corrections are listed below.

- Figure 11. The data shown in (a) and (b) of this figure is mismatched. The pattern in (a) is supposed to result in the behavior in (b), but actually it does not and such behavior would be inconsistent. Fortunately, this does not affect the analysis because (a) still corresponds to an arm motion that follows the target in a way similar to (b) and others shown in the paper.

The online version of the original article can be found under doi:10.1007/s10710-007-9044-8.

\section{S. Gotshall $(\bowtie) \cdot$ T. Soule}

Department of Computer Science, University of Idaho, Moscow, ID, USA

e-mail: stanleyg@vandals.uidaho.edu

T. Soule

e-mail: tsoule@cs.uidaho.edu

\section{K. Browder}

Department of Health, Physical Education, Recreation, and Dance, University of Idaho,

Moscow, ID, USA

e-mail: kbrowder@uidaho.edu

\section{J. Sampson}

Department of Mechanical Engineering, University of Idaho, Moscow, ID, USA

e-mail: samp7553@uidaho.edu

R. Wells

Department of Electrical and Computer Engineering, University of Idaho, Moscow, ID, USA

e-mail: rwells@mrc.uidaho.edu 
- Equation 5 has errors in the 2 nd and 3 rd term. It should read:

$$
\begin{aligned}
v(t)= & \Omega(t-1) v(t-1)+\left(c_{1}\right)\left(r_{1}\right)\left(x_{b}-x(t-1)\right) \\
& +\left(c_{2}\right)\left(r_{2}\right)\left(x_{g b}-x(t-1)\right) .
\end{aligned}
$$

The $x$ component is currently missing which is a key component of the algorithm.

- A sentence in section 4.4 (the neural net modeling section) was omitted during editing. It is the following: "Both afferent neurons' outgoing excitatory synaptic weights are allowed three times the value of the other neurons' outgoing weights to have an effect comparable to the remaining inputs to the alpha-MN." Given the other model parameters, it is unlikely this would prevent the model from generating the behavior types highlighted in the paper; however, the afferent pathways are key features of the model with respect to the observed behaviors and this variation may influence how readily the training algorithms take advantage of these pathways. 